\title{
Radio Science
}

\section{RESEARCH ARTICLE \\ 10.1002/2016RS006178 \\ Internal Natural Resonances Prediction Through the Theory of Characteristic Modes}

Key Points:

- An interpretation of the resonances provided by the theory of characteristic modes on a dielectric cylinder is carried out

- Internal and external characteristic mode resonances provide only an approximation to internal natural resonances, not external

- Prediction of internal natural resonances by characteristic mode resonances becomes more inaccurate as relative permittivity decreases

Correspondence to:

T. Bernabeu-Jimenez,

toberji@gmail.com

\section{Citation:}

Bernabeu-Jimenez, T.,

Valero-Nogueira, A., Vico-Bondia, F.,

\& Kishk, A. A. (2018). Internal natural resonances prediction through the theory of characteristic modes. Radio Science, 53, 397-405. https://doi.org/10.1002/2016RS006178

Received 30 SEP 2016 Accepted 8 FEB 2018 Accepted article online 20 FEB 2018 Published online 6 APR 2018

\author{
Tomas Bernabeu-Jimenez ${ }^{1}$ (D), Alejandro Valero-Nogueira ${ }^{1}$, Felipe Vico-Bondia ${ }^{1}$, \\ and Ahmed A. Kishk ${ }^{2}$ \\ ${ }^{1}$ Departamento de Comunicaciones, Universitat Politècnica de València, Valencia, Spain, ${ }^{2}$ Department of Electrical \\ and Computer Engineering, Concordia University, Montreal, Quebec, Canada
}

\begin{abstract}
Some aspects in the interpretation of the resonances provided by the theory of characteristic modes for dielectric bodies are analyzed. The analysis has been performed analytically based on the Poggio-Miller-Chang-Harrington-Wu-Tsai formulation on a canonical problem, the infinite dielectric circular cylinder. First, natural resonances and characteristic mode resonances are presented and compared. It has been observed that characteristic mode resonances are in general near to internal natural resonances but not to external ones. It is also demonstrated that characteristic resonances become closer to internal natural ones as the relative permittivity of the dielectric cylinder is increased.
\end{abstract}

\section{Introduction}

Natural resonances (NRs) on dielectric bodies have been extensively studied in the past. Some of the main geometries studied are the infinite circular cylinder and the sphere. The infinite circular cylinder was first studied by Rayleigh (1918) and the sphere by Mie (1908). After these two works, many works have been carried out to understand physically the behavior of the electromagnetic fields inside and outside of them. Linked to this, many of these works treated the resonance problem of natural modes that can be excited by a source.

For example, with regard to the infinite dielectric circular cylinder, in Van Bladel (1977), resonant modes, field patterns, resonant frequencies, and quality factors of the modes were determined. In Dettmann et al. (2009) internal and external resonant modes for both $T M^{z}$ and $T E^{z}$ polarizations were analyzed providing a mathematical procedure for singling out internal and external NRs without resorting to a priori visualization of the electromagnetic field of the mode. Even if simple, the analysis of an infinite dielectric circular cylinder may be of practical use in problems such as the estimate of cylinder radius for optical-communications fibers through its transverse resonances (Kotlyar et al., 2016; Owen et al., 1981, among others).

Concerning the dielectric sphere, in Conwell et al. (1984) natural resonant frequencies and poles associated with the electromagnetic modes were analyzed. In C.-C. Chen (1998), internal and external NRs were exhaustively studied via the Mie series, and in Lee et al. (2013), the Cauchy method was applied to extract the NRs of dielectric spheres considering different parameters such as permittivity and conductivity.

The knowledge of these two canonical geometries has been very important, not only for its own sake but also for the insight it provides for other more general geometries. That is why new methods have been developed to extract NRs from arbitrary structures, such as the Cauchy method mentioned above. Other methods are the singularity expansion method (Baum, 2012) and the Prony's method, among others. An alternative approach, similar to singularity expansion method, would be to determine the NRs by searching the complex frequency plane for the zeros of the determinant of the method of moments matrix, (Glisson et al., 1983; Kishk et al., 1993). All the methods mentioned provide the same set of resonances.

Unlike this type of resonances, another method is becoming very popular to find other type of modes and resonances. This method is known as Characteristic Mode Analysis (CMA). When compared to NRs, whose resonances are generally complex, the resonances provided by CMA are all real. CMA was first proposed by Garbacz (1968) and reformulated by Harrington, Mautz, and Chang, establishing a more direct method to obtain Garbacz's modal expansion (Chang \& Harrington, 1977; Harrington \& Mautz, 1971; Harrington et al., 1972). They considered integro-differential formulations using the impedance matrix of the method of moments. Although many design-related works have been published since then, particularly devoted 
to antennas and scatterers, there are still theoretical aspects that are unknown and need to be clarified to provide a correct interpretation of the characteristic mode solutions. In order to interpret these solutions, it is interesting to use NRs as a reference, since NRs are unique, regardless of the method considered to obtain them and the integral formulation approach used (Larison, 1989). It is worth mentioning Sarkar et al. (2016), where some insightful discussions about characteristic mode resonances (CMRs) and NRs for conducting bodies led to greater clarity on this subject. However, with regard to dielectric bodies, there are still many issues to be clarified because the formulation derived in Chang and Harrington (1974), if hastily interpreted, may lead to these misunderstandings. In Alroughani (2013) it is concluded that NRs and CMRs are very close to each other. Moreover, they found some spurious modes called nonphysical modes. On the other hand, in Bernabeu-Jiménez et al. (2015) it is concluded that NRs and CMRs are, in fact, very different. This leads to opposite views. To explicitly determine CMRs on dielectric bodies, Y. Chen and Wang $(2015,2014)$ proposed an alternative generalized eigenvalue equation involving only the equivalent electric current on the surface. Starting from the Poggio-Miller-Chang-Harrington-Wu-Tsai (PMCHWT) operator, they obtained a modified matrix operator to form a new generalized eigenvalue equation from which CMs were calculated for dielectric bodies. Furthermore, in Hu and Wang (2016), five integral equation formulations were compared for CMA of dielectric resonators and concluded that PMCHWT cannot fully predict resonant frequency of dielectric resonators.

In this paper, the conclusions drawn by Alroughani (2013) and Bernabeu-Jiménez et al. (2015) will be discussed and linked throughout the paper. In addition, some aspects in the interpretation of the resonances provided by CMA are analyzed. This has been done using the analytical solution of the PMCHWT integro-differential operator for the infinite dielectric circular cylinder. First, NRs and CMRs are presented and compared. Second, it is demonstrated that the resonant frequencies of the CMRs are only close to the resonant frequencies of the internal NRs and do not provide information at all about the external ones. Finally, it is shown that the resonant frequencies of the CMRs become closer to the resonant frequencies of the internal NRs as the relative permittivity of the dielectric cylinder is increased. Furthermore, it is also found that, for a given permittivity, each natural mode observed provides a different error between the CMRs and the NRs.

\section{Brief Introduction to the PMCHWT Formulation and Its NRs and CMRs}

Consider a dielectric body illuminated by an incident electric and magnetic field, $\mathbf{E}^{\text {inc }}$ and $\mathbf{H}^{\text {inc }}$, respectively. These incident fields induce electric and magnetic equivalent surface currents $\mathbf{J}$ and $\mathbf{M}$ flowing on the dielectric surface that radiates the scattered fields $\mathbf{E}^{s}$ and $\mathbf{H}^{s}$. After imposing the boundary conditions for the tangential field on the dielectric surface, the problem to be solved can be written from a functional viewpoint defined by the PMCHWT formulation as

$$
\left[\begin{array}{cc}
L_{e} & -C \\
C & L_{m}
\end{array}\right]\left[\begin{array}{c}
\mathbf{J} \\
\mathbf{M}
\end{array}\right]_{\tan }=\left[\begin{array}{l}
\mathbf{E}^{\text {inc }} \\
\mathbf{H}^{\text {inc }}
\end{array}\right]_{\tan }
$$

where $L_{e}, L_{m}$, and $C$ are integro-differential operators defined in Chang and Harrington (1974). Equation (1) can be also expressed as follows:

$$
T(\mathbf{f})=\mathbf{g}^{\text {inc }},
$$

where the subscript "tan" have been dropped for brevity. Moreover,

$$
T=\left[\begin{array}{cc}
L_{e} & -C \\
C & L_{m}
\end{array}\right] ; \quad \mathbf{f}=\left[\begin{array}{c}
\mathbf{J} \\
\mathbf{M}
\end{array}\right] ; \quad \mathbf{g}^{\mathrm{inc}}=\left[\begin{array}{c}
\mathbf{E}^{\mathrm{inc}} \\
\mathbf{H}^{\text {inc }}
\end{array}\right],
$$

being $T$ the operator tangent to the surface of the object, $\mathbf{f}$ the current vector, and $\mathbf{g}^{\text {inc }}$ the incident field vector.

The resonance problem considered as NR problem is formulated in (4), where no excitation is applied, that is, $\mathbf{g}^{\text {inc }}=0$. Equation (4) defines the natural frequencies and natural modes of the target involved in the scattering process for dielectric bodies:

$$
T(\mathbf{f}(\omega))=0 .
$$

This means that one seeks for the nontrivial solutions of (4), which requires to find the solutions in which $T$ is a singular operator. This generally occurs at an infinite number of discrete complex frequencies, known 
as natural frequencies, $\omega=\omega^{\prime}+j \omega^{\prime \prime}$, where $\omega^{\prime}$ provides the natural resonant frequency of a given natural mode and $\omega^{\prime \prime}$ its damping factor. The damping factor is linked to the radiating properties of a given mode (C.-C. Chen, 1998).

To find the singularities of the $T$ operator, one can seek for the zeros of the eigenvalues of the standard eigenvalue problem (5), $v_{n}=0$ or, equivalently, to solve for $\operatorname{det}(T)=0$, being $T$ a matrix operator, since $\operatorname{det}(T)=\prod_{n} v_{n}$.

$$
T \mathbf{f}_{n}(\omega)=v_{n} \mathbf{f}_{n}(\omega)
$$

In (5), $\mathbf{f}_{n}$ is the natural basis or the natural modes for the PMCHWT formulation. It is important to note that both $\mathbf{f}_{n}$ and $v_{n}$ are complex valued.

On the other hand, the resonance problem considered as CMR problem is formulated in (7). But we must first consider a symmetrization of the $T$ operator presented in (6), renamed as $T^{\prime}$ and the changes produced in $\mathbf{f}$ and $\mathbf{g}$ as it is expressed below:

$$
T^{\prime}=\left[\begin{array}{cc}
L_{e} & -j C \\
-j C & L_{m}
\end{array}\right] ; \quad \mathbf{f} \prime=\left[\begin{array}{c}
\mathbf{J} \\
j \mathbf{M}
\end{array}\right] ; \quad \mathbf{g} \prime^{\text {inc }}=\left[\begin{array}{c}
\mathbf{E}^{\mathrm{inc}} \\
j \mathbf{H}^{\mathrm{inc}}
\end{array}\right]
$$

These changes were first proposed by Chang and Harrington (1974). And they were considered because in the generalized eigenvalue problem governing the theory of characteristic modes (7), $T_{2}^{\prime}$ and $T_{1}^{\prime}$ have to be Hermitian operators to obtain both eigenvalues $\lambda_{n}$ and eigenvectors $\mathbf{f} \prime_{n}$ real valued. Here is the importance of theory of characteristic modes solutions.

$$
\left[T_{2}^{\prime}\right]\left[\mathbf{f} \prime_{n}\right]=\lambda_{n}\left[T_{1}^{\prime}\right]\left[\mathbf{f} \prime_{n}\right]
$$

In equation (7) $T_{2}^{\prime}$ and $T_{1}^{\prime}$ are the imaginary and the real parts of the symmetrized PMCHWT operator $\left(T^{\prime}=T_{1}^{\prime}+\right.$ $\left.j T_{2}^{\prime}\right)$, respectively. $\mathbf{f} \prime_{n}$ are the characteristic currents, and $\lambda_{n}$ are their corresponding characteristic eigenvalues.

In the following, we will ignore the primed variables, and we will calculate the NRs from the determinant of the symmetric operator $T^{\prime}$, since to make the determinant of the symmetric operator $T^{\prime}$ (6), or by using the antisymmetric operator $T$ (3), the same result is obtained. Thus, we will recall $T^{\prime}$ as $T$.

\section{Infinite Dielectric Circular Cylinder}

In this section, NRs and CMRs are compared through the PMCHWT formulation. Since we are dealing with an infinite circular cylinder, the PMCHWT operator can be written in its closed form for both the $T M^{z}$ and the $T E^{Z}$ polarizations. Inasmuch as the scope of this paper is to show the differences between both types of resonances, the calculation procedure of these operators is not included here. Chang and Harrington (1974) was followed to derive these analytical operators.

Equations (8) and (9) represent the analytical $T M_{n}^{z}$ and $T E_{n}^{z}$ PMCHWT matrix operators, where $a$ is the cylinder radius, $H_{n}^{(2)}(x)$ are the Hankel functions of the second kind, $J_{n}(x)$ the Bessel functions of the first kind, $n$ the azimuthal mode order, $k_{j}$ the wave number, and the pairs $\left(\epsilon_{j}, \mu_{j}\right)$ are the permittivity and the permeability, respectively. The primes on the Bessel and Hankel functions denote differentiation with respect to the entire argument. The subscripts $j=1$ or $j=2$ stands for outer and inner media, respectively.

$$
\begin{aligned}
& T_{n}^{T M^{2}}=(\pi a)^{2}\left[\begin{array}{ccc}
\omega\left(\mu_{2} J_{n}\left(k_{2} a\right) H_{n}^{(2)}\left(k_{2} a\right)+\mu_{1} J_{n}\left(k_{1} a\right) H_{n}^{(2)}\left(k_{1} a\right)\right) & \left(k_{2} J_{n \prime}\left(k_{2} a\right) H_{n}^{(2)}\left(k_{2} a\right)+k_{1} J_{n}\left(k_{1} a\right) H_{n \prime}^{(2)}\left(k_{1} a\right)\right) \\
\left(k_{2} J_{n \prime}\left(k_{2} a\right) H_{n}^{(2)}\left(k_{2} a\right)+k_{1} J_{n}\left(k_{1} a\right) H_{n \prime}^{(2)}\left(k_{1} a\right)\right) & \omega\left(\epsilon_{2} J_{n \prime}\left(k_{2} a\right) H_{n \prime}^{(2)}\left(k_{2} a\right)+\epsilon_{1} J_{n \prime}\left(k_{1} a\right) H_{n \prime}^{(2)}\left(k_{1} a\right)\right)
\end{array}\right] \\
& T_{n}^{T E^{z}}=(\pi a)^{2}\left[\begin{array}{ccc}
\omega\left(\mu_{2} J_{n \prime}\left(k_{2} a\right) H_{n \prime}^{(2)}\left(k_{2} a\right)+\mu_{1} J_{n \prime}\left(k_{1} a\right) H_{n \prime}^{(2)}\left(k_{1} a\right)\right) & \left(k_{2} J_{n \prime}\left(k_{2} a\right) H_{n}^{(2)}\left(k_{2} a\right)+k_{1} J_{n}\left(k_{1} a\right) H_{n \prime}^{(2)}\left(k_{1} a\right)\right) \\
\left(k_{2} J_{n \prime}\left(k_{2} a\right) H_{n}^{(2)}\left(k_{2} a\right)+k_{1} J_{n}\left(k_{1} a\right) H_{n \prime}^{(2)}\left(k_{1} a\right)\right) & \omega\left(\epsilon_{2} J_{n}\left(k_{2} a\right) H_{n}^{(2)}\left(k_{2} a\right)+\epsilon_{1} J_{n}\left(k_{1} a\right) H_{n}^{(2)}\left(k_{1} a\right)\right) .
\end{array}\right]
\end{aligned}
$$

\subsection{Natural Resonances}

As previously introduced, NRs are extracted from the zeros of the determinant of the PMCHWT matrix operator (Glisson et al., 1983). Thus, concerning the infinite dielectric circular cylinder, the determinant of the operators (8) and (9) have to be equal to 0 to find its corresponding NRs. The cylinder under study is $\epsilon_{r_{2}}=9$ and $\mu_{r_{2}}=1$, 


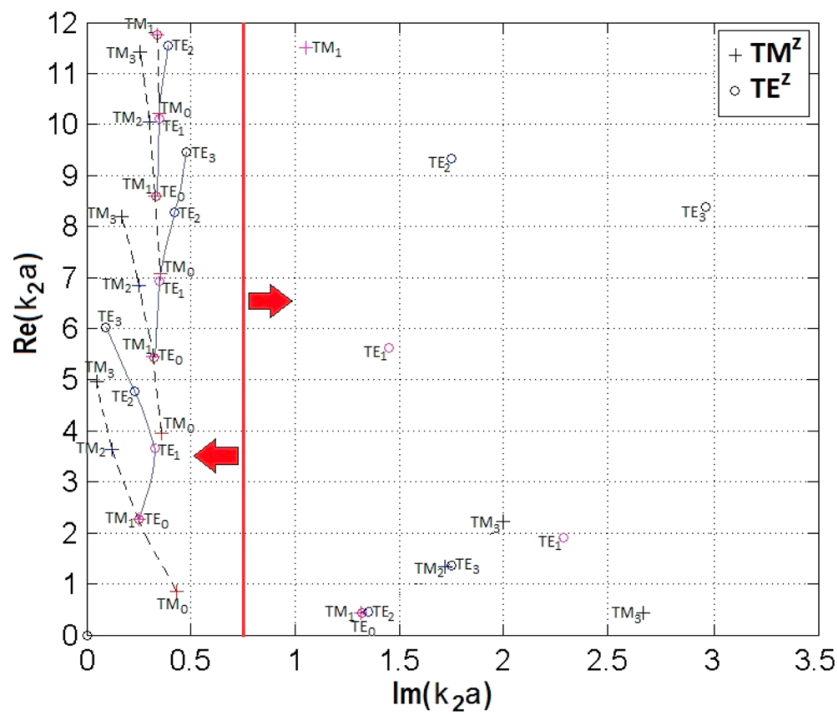

Figure 1. Natural resonances (NRs) obtained from the Poggio-Miller-ChangHarrington-Wu-Tsai operators (8) and (9) and NRs obtained from the scattering coefficient of the harmonic series solution (16) and (17). Both procedures give the same results and therefore coincide each other in the graph. Thus, without making any difference between them, only NRs for the TM and TE modes have been plotted. Placement of dividing vertical line at $\operatorname{Im}\left(k_{2} a\right)=0.75$ is arbitrary, with the only purpose of indicing where the internal/external NRs are located. Internal NRs are located to the left of the line, and external NRs to the right. The relative permittivity of the cylinder is $\epsilon_{2}=9$, and the outer media is considered vacuum. and the surrounding media is considered to be vacuum. Substituting this values in (8) and (9), we can find some of the first natural resonances of the $T E_{n}^{z}$ and $T M_{n}^{z}$ modes for $n=0,1,2,3$. These resonances are shown in Figure 1.

In order to determine the zeros of $\operatorname{det}\left(T_{n}\left(k_{2} a\right)\right)$, a standard zero-finding routine is used as in Glisson et al. (1983).

These NRs are located in the $k_{2} a$ complex plane as done in C.-C. Chen (1998) for the dielectric sphere. $k_{2} a=\omega \sqrt{\epsilon_{2} \mu_{2}}, \epsilon_{2}$ and $\mu_{2}$ are the wave number, permittivity, and permeability of the inner media. The subscript " 2 " designates the inner media. In Figure $1 T M_{n}^{z}$ and $T E_{n}^{z}$ modes are marked with "+" and "॰," respectively. The real part of the NRs provides the resonant frequency and the imaginary part the damping factor. The damping factor provides information on how a given mode is leaking.

Considering the conclusions presented in C.-C. Chen (1998), the same can be concluded here: the NRs can be separated into internal and external. The internal NRs are caused by the internal waves that experience multiple internal reflections, whereas the external NRs are caused by creeping waves. These creeping waves propagate along the surface with attenuation due to the continuous radiation in tangent direction. In Figure 1, the internal NRs are on the left-hand side of a vertical line, and the external NRs are located on the right-hand side, both indicated by arrows. The vertical line only serves to divide the graph between the internal and the external NRs, helping the graph understanding. In order to differentiate between internal and external NRs, one can use the formulas given by Dettmann et al. (2009), where a complete analysis for an infinite dielectric circular cylinder is shown to clearly distinguish between internal and external modes for both $T M^{z}$ and $T E^{z}$ polarizations. For the $T M^{z}$ polarization, the internal and external NRs can be found using the limits (10)-(12) and (13), respectively:

$$
\begin{gathered}
\lim _{\epsilon_{r_{2}} \rightarrow \infty}\left[k_{2} a\right]_{n, m}=j_{n-1, m} \Rightarrow n \neq 0 \\
\lim _{\epsilon_{r_{2}} \rightarrow \infty}\left[k_{2} a\right]_{0, m}=j_{1, m-1} \Rightarrow m \neq 1 \\
\lim _{\epsilon_{r_{2}} \rightarrow \infty}\left[k_{2} a\right]_{0,1}=0 \\
\lim _{\epsilon_{r_{2}} \rightarrow \infty}\left[k_{1} a\right]_{n, m}=h_{n, m} .
\end{gathered}
$$

Regarding the $T E^{Z}$ polarization, the internal and external NRs can be found using equations (14) and (15), respectively.

$$
\begin{gathered}
\lim _{\epsilon_{r_{2}} \rightarrow \infty}\left[k_{2} a\right]_{n, m}=j_{n, m} \\
\lim _{\epsilon_{r_{2}} \rightarrow \infty}\left[k_{1} a\right]_{n, m}=h_{n, m}^{\prime},
\end{gathered}
$$

where the subscripts $n$ and $m$ define the $m$ th resonance of the $n$ th-order Bessel and Hankel functions. These equations provide the difference between internal and external NRs. When the relative permittivity tends to infinity, internal NRs, which start being complex valued, become real in the limit according to the corresponding definition. On the contrary, external NRs remain complex valued. This allows one to distinguish between internal and external NRs. 


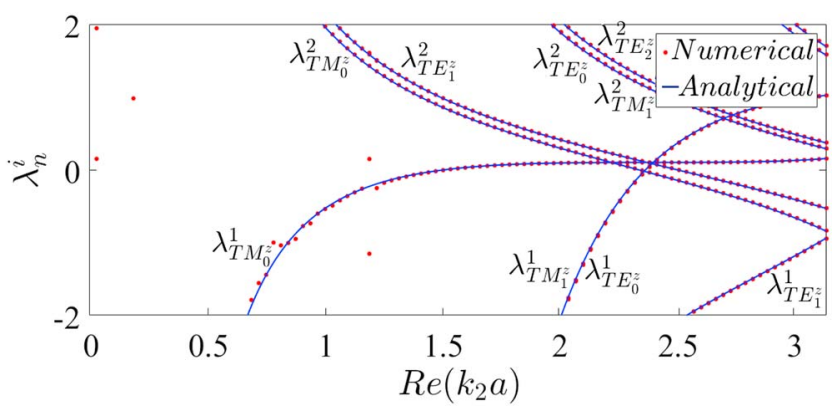

Figure 2. Comparison between analytic and numerical characteristic eigenvalues for the infinite dielectric circular cylinder. The relative permittivity of the cylinder is $\epsilon_{r_{2}}=9$, and the outer media is considered vacuum.

To verify the correctness of equations (8) and (9), a comparison between the scattering coefficient poles of the harmonic series solution (16) and (17) is performed in Figure 1. In Figure 1, NRs obtained from both procedures coincide each other.

$$
\begin{aligned}
& T M_{z} \rightarrow \eta_{2} J_{n}\left(k_{2} a\right) H_{n \prime}^{(2)}\left(k_{1} a\right)-\eta_{1} J_{n \prime}\left(k_{2} a\right) H_{n}^{(2)}\left(k_{1} a\right)=0 \\
& T E_{z} \rightarrow \eta_{2} J_{n \prime}\left(k_{2} a\right) H_{n}^{(2)}\left(k_{1} a\right)-\eta_{1} J_{n}\left(k_{2} a\right) H_{n \prime}^{(2)}\left(k_{1} a\right)=0 .
\end{aligned}
$$

\subsection{Characteristic Mode Resonances}

CMRs are obtained from the following generalized eigenvalue problem (7). By definition, the eigencurrents and the eigenvalues are both real, and CMRs are obtained from the eigenvalues $\lambda_{n}$. When $\lambda_{n}= \pm \infty$ (asymptotic behavior) or $\lambda_{n}=0$ means that at those frequencies there is a suspected internal or external CMRs, respectively. As in the case of NRs, CMRs are also classified in two types, internal and external. Nevertheless, as we will see in the next subsection, they are different from NRs.

Concerning the infinite dielectric cylinder, characteristic eigenvalues can be found substituting equations (8) and (9) in (7). Let us consider the same example as for the previous section: a nonmagnetic homogeneous cylinder with $\epsilon_{r_{2}}=9$. The characteristic eigenvalues obtained are shown in Figure 2, in which the analytical eigenvalues are validated by comparing them with those obtained numerically by an in-house code interfaced with (FEKO Suite 7.0). Notice that a one-dimensional periodic boundary condition and the surface equivalence principle were used to simulate the infinite cylinder with FEKO.

Figure 2 shows an excellent agreement between the numerical and the analytical solutions. Furthermore, as shown in Figure 2, two eigenvalues are obtained per each mode, as it must be, given that the PMCHWT operator is a 2-by-2 linear integro-differential operator. In Figure 2, the eigenvalues with superscripts 1 and 2 are referred to as the physical and the nonphysical characteristic eigenvalues, respectively. This notation was adopted after the publication (Alroughani, 2013), where a more detailed explanation about nonphysical characteristic modes can be seen.

In addition, each eigenvalue has its associated subscript indicating the polarization type and the order of the cylindrical harmonic solution, that is, $T M_{n^{\prime}}^{z} T E_{n}^{z}$. It is interesting to note that eigenvalues corresponding to the $T E_{0}^{z}$ and the $T M_{1}^{z}$ coincide with each other. Now turning to the nonphysical modes, this term was first introduced by Alroughani et al. (2014), since they found that certain types of eigenvalue solutions provided

Table 1

NRs for the First and Second Modes $(m=1,2)$, Poles $(n=0,1,2)$, for the Infinite Dielectric Circular Cylinder Graphed in Figure 1

\begin{tabular}{lcccc}
\hline$k_{2} a$ & $\mathrm{NRs} m=1$ & \multicolumn{3}{c}{$\mathrm{NRs} m=2$} \\
\hline$n$ & $T M_{n, 1}^{z}$ & $T E_{n, 1}^{z}$ & $T M_{n, 2}^{z}$ & $T E_{n, 2}^{z}$ \\
0 & $0.85+\mathrm{j} 0.43$ & $2.27+\mathrm{j} 0.25$ & $3.95+\mathrm{j} 0.36$ & $5.44+\mathrm{j} 0.33$ \\
1 & $2.27+\mathrm{j} 0.25$ & $3.65+\mathrm{j} 0.33$ & $5.45+\mathrm{j} 0.32$ & $6.93+\mathrm{j} 0.35$ \\
2 & $3.64+\mathrm{j} 0.12$ & $4.78+\mathrm{j} 0.23$ & $6.84+\mathrm{j} 0.25$ & $8.27+\mathrm{j} 0.42$ \\
\hline
\end{tabular}


Table 2

External Characteristic Mode Resonant Frequencies for the Infinite Dielectric Circular Cylinder Obtained From $\lambda_{T M_{n}^{z}}^{1} \lambda_{T M_{n}^{z}}^{2} \lambda_{T E_{n}^{z}}^{1}$ and $\lambda_{T E_{n}^{z}}^{2}$ Curves in Figure2

\begin{tabular}{lcccc}
\hline $\operatorname{Re}\left(k_{2} a\right)$ & $\mathrm{CMRs} \lambda_{X}^{1}$ & \multicolumn{3}{c}{$\mathrm{CMRs} \lambda_{X}^{2}$} \\
\hline$n \backslash X$ & $T M_{n, 1}^{z}$ & $T E_{n, 1}^{z}$ & $T M_{n, 1}^{z}$ & $T E_{n, 1}^{Z}$ \\
0 & 1.514 & 2.371 & 2.340 & 3.490 \\
1 & 2.371 & 3.545 & 3.490 & 2.505 \\
\hline
\end{tabular}

Note. These values correspond to the zero crossings of the eigenvalue curves.

by the PMCHWT operator diagonalization did not satisfy the orthogonality far field relation presented by Chang and Harrington (1974). Because the nonphysical characteristic modes were declared of no relevance, the next section only deals with resonances of the physical characteristic modes.

\subsection{NRs Versus CMRs}

In this subsection, the study concerns mainly with the differences between NRs and CMRs. NRs were also compared with CMRs for DRs in Alroughani (2013) and Bernabeu-Jiménez et al. (2015). In Alroughani (2013) an isolated cylindrical dielectric resonator with $\epsilon_{r}=79.7$ was studied. They concluded that the CMRs occur at the same resonant frequencies as the NRs with an agreement between the two sets of frequencies within 4\%. However, in Bernabeu-Jiménez et al. (2015), an infinite dielectric circular cylinder with $\epsilon_{r_{2}}=9$ was analytically analyzed concluding that the resonant frequencies of the CMRs were different from the NRs, observing a larger difference than in Alroughani (2013) and stating that CMR can be seen as a different type of resonances. It is believed that the reason that Alroughani (2013) arrived at a different conclusion was probably because a high relative permittivity value was used. Here, however, it is observed that when lower relative permittivities are considered, the differences between the resonant frequencies of CMRs and NRs are much larger. The results presented in this paper are therefore crucial to the application of CMA to DRs and dielectric resonator antenna design.

The statements below will be demonstrated regarding the infinite dielectric circular cylinder:

1. CMRs are distinct from NRs.

2. The resonant frequencies provided by internal $\left(\lambda_{n} \rightarrow \pm \infty\right)$ and external $\left(\lambda_{n}=0\right)$ CMRs provide values that are close to the resonant frequencies of internal NRs only, while the resonant frequencies of external NRs cannot be found by the resonant frequencies of the CMRs.
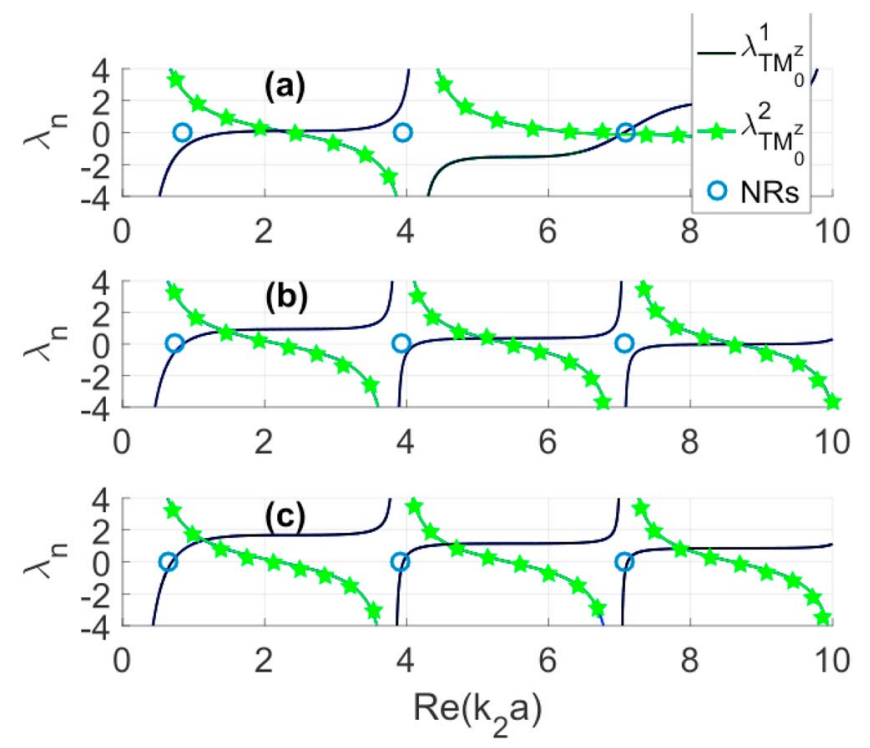

Figure 3. A comparison between the characteristic eigenvalues and internal natural resonances $\left(T M_{01}^{z}, T M_{02}^{z}\right.$, and $T M_{03}^{z}$ circles from left to right, respectively) considering relative permittivity for the dielectric cylinder of $\epsilon_{r_{2}}=9$ in (a), $\epsilon_{r_{2}}=90$ in (b), and $\epsilon_{r_{2}}=900$ in (c). 


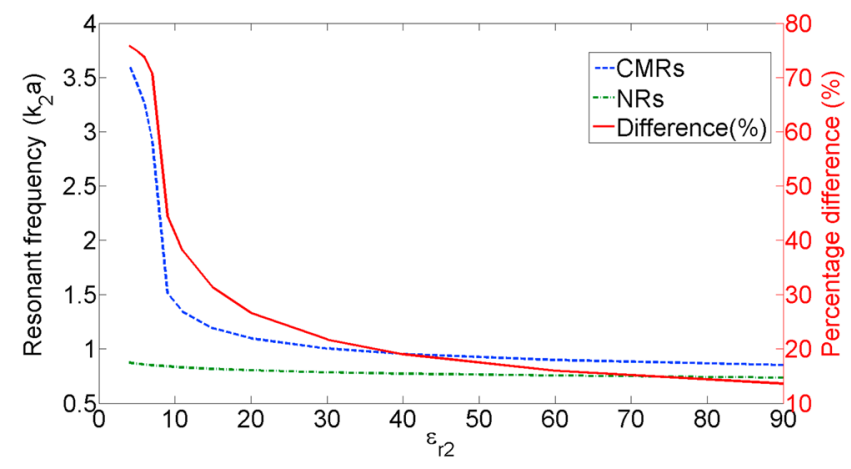

Figure 4. Comparison between the resonant frequencies of the characteristic mode resonances (CMRs) and natural resonances (NRs) versus $\epsilon_{r_{2}}$ for $T M^{z}(n=0)$ mode.

3. As the dielectric permittivity increase, the resonant frequencies of the internal NRs are closer to the resonant frequencies of the both internal and external CMRs.

4. The gap found between CMRs and internal NRs differs depending on the electromagnetic mode considered. In other words, each characteristic mode differs from its corresponding natural mode in a different way when it is subjected to permittivity variations and is compared with other modes having different order.

To demonstrate that NRs are different from CMRs, Tables 1 and 2 show some of the resonances obtained by both procedures and plotted in Figures 1 and 2, respectively. It is obvious that the real part of the NRs are completely different from their corresponding CMRs.

It is interesting to note that, unlike a shielded cavity, whose internal NRs are located on the real axis of frequencies, a dielectric cavity has its internal NRs on the complex frequency plane because their corresponding internal modes are leaking. This leakage is due to radiation losses through the cavity wall. For example, an infinite perfect electric conductor circular cylinder exhibits its internal NRs at frequencies that meet conditions $J_{n}(k a)=0\left(T M_{n}^{z}\right)$ and $J_{n}^{\prime}(k a)=0\left(T E_{n}^{z}\right)$, resulting in real frequencies, not complex numbers. This can be seen from Sarkar et al. (2016) where a thorough discussion regarding internal NRs and CMRs for a perfect electric conductor circular cylinder was carried out.

As can be seen from Table 1, the dielectric cylinder has complex-valued internal NRs. On the other hand, we note that CMRs are represented on the real $k_{2} a$ axis. Let us consider, for instance, $T M_{0}^{z}$ mode as in Bernabeu-Jiménez et al. (2015) to check the closeness between the resonant frequencies of CMRs and NRs, and the possible relation between them. Figure 3 shows characteristic mode eigenvalues as curves and NRs as circles (only internal). Recall that external CMRs occur for those frequencies where $\lambda_{T M_{0}^{z}}^{1}=0$, while the internal ones are occurring for those frequencies where $\lambda_{T M_{0}^{2}}^{1} \rightarrow \pm \infty$. Values are shown for three relative permittivity values of $\epsilon_{r_{2}}=9$ in (a), $\epsilon_{r_{2}}=90$ in (b), and $\epsilon_{r_{2}}=900$ in (c).

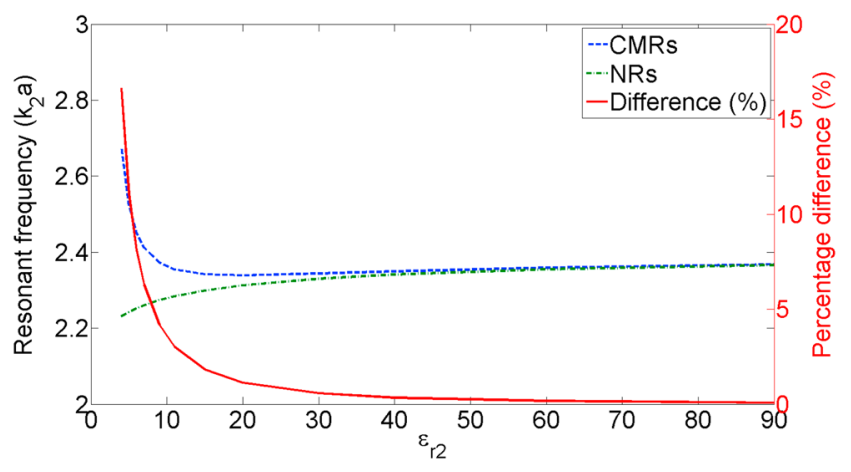

Figure 5. Comparison between the resonant frequencies of the characteristic mode resonances (CMRs) and the natural resonances (NRs) versus $\epsilon_{r_{2}}$ for the $T E^{Z}(n=0)$ mode.
From left to right, the blue circles correspond to the projection of the internal NRs frequencies on the real $k_{2} a$ axis for $T M_{01}^{z}, T M_{02}^{z}$, and $T M_{03}^{z}$ poles, respectively. It is evident that depending on the relative permittivity used, the resonant frequencies of the internal NRs can be more or less predicted from the characteristic eigenvalues $\lambda_{T M_{0}^{z}}^{1}$. The higher the relative permittivity, the closer the resonant frequencies of the CMRs are to the NRs. This occurs because internal NRs are in different imaginary planes, $\operatorname{Im}\left(k_{2} a\right) \neq 0$, for each permittivity, while CMRs are always located on $\operatorname{Im}\left(k_{2} a\right)=0$. It can be concluded then that the resonant frequencies of the CMRs are relatively close to the internal NRs depending on the permittivity. The same applies to the radar cross section spectrum, which also provides values that are close to NRs (Moser et al., 1989). Therefore, although they are different, internal NRs are more predictable as permittivity increases.

To end the demonstration on whether the frequencies of the CMRs and internal NRs become closer as the relative permittivity increases, Figures 4 and 5 show the percentage variation between CMRs and the real part 


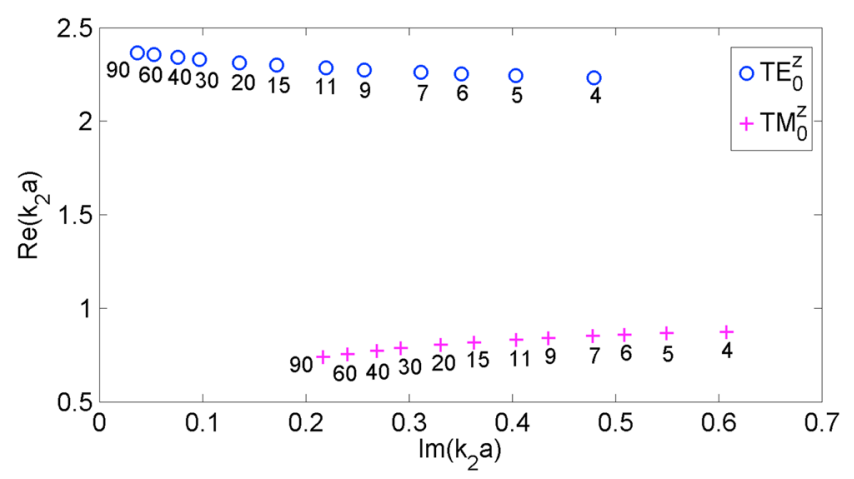

Figure 6. Displacement of the natural resonances due to the variation of the permittivity for the $T M^{z}$ and $T E^{Z}(n=0)$ mode. Numbers below the poles are its corresponding $\epsilon_{r_{2}}$. of NRs. Figure 4 shows the percentage difference between the two types of resonant frequencies for the $T M_{n}^{z}(n=0)$. As permittivity decreases, the percentage difference increases significantly. The same applies to Figure 5, where $T E_{n}^{Z}(n=0)$ mode behavior is shown. The percentage difference for $T E_{n}^{z}$ mode is less significant than for $T M_{n}^{z}$ mode because $T E_{n}^{z}$ mode is located closer to $\operatorname{Re}\left(k_{2} a\right)$ axis than $T M_{n}^{z}$ is; that is, the damping is lower. This can be seen in Figure 1.

Why is this happening? The answer is simply because the higher the permittivity of the dielectric body, the closer to the $\operatorname{Re}\left(k_{2} a\right)$ axis the internal resonances are, as explained by equations (10)-(15) above. As a last evidence in this regard, Figure 6 shows how $T M_{n}^{z}$ and $T E_{n}^{z}(n=0)$ NRs move into the complex $k_{2} a$ plane as $\epsilon_{r_{2}}$ decreases. Number next to each pole in Figure 6 is the corresponding $\epsilon_{r_{2}}$ value. As $\epsilon_{r_{2}}$ decreases, the damping becomes higher and so does radiation.

\section{Acknowledgments}

This work was supported by the Spanish Ministerio de Economía y Competitividad under the project TEC2013-47360-C3-3P. This paper is a theoretical research article and contains no data.

\section{Conclusion}

In summary, it has been shown that CMRs are different from NRs. At most, and depending on the relative permittivity considered, the resonant frequencies of the CMRs might be used as a first guess in an internal NRs search in the complex frequency plane. In addition, the resonant frequencies of the CMRs only predict internal the resonant frequencies of the NRs because of their proximity to the $\operatorname{Re}\left(k_{2} a\right)$ axis. It has been also demonstrated that the differences between the resonant frequencies of the CMRs and internal NRs depend on relative permittivity. Large $\epsilon_{r_{2}}$ provide closer values. It has been concluded that this is due to a decrease in the dumping factor of a given particular mode. Besides, it has been observed that the difference between both CMRs and internal NRs also depends on the particular electromagnetic mode considered.

The analysis has been performed on an infinite dielectric circular cylinder. It provides the accurate analytical solutions required to establish reliable observations. Notice that subtle resonant frequency differences between the two families of modes have led other authors to misinterpretations.

\section{References}

Alroughani, H. (2013). An appraisal of the characteristic modes of composite objects (Master thesis). University of Ottawa.

Alroughani, H., Ethier, J. L., \& McNamara, D. (2014). Observations on computational outcomes for the characteristic modes of dielectric objects. In Antennas and Propagation Society International Symposium (APSURSI), 2014 IEEE (pp. 844-845). IEEE.

Baum, C. E. (2012). The singularity expansion method in electromagnetics: $A$ summary survey and open questions. Albuquerque, NM: SUMMA Foundation. Lulu.com.

Bernabeu-Jiménez, T., Valero-Nogueira, A., Vico-Bondia, F., \& Kishk, A. A. (2015). Relation between characteristic modes and complex natural resonances. In Antennas and Propagation \& USNC/URSI National Radio Science Meeting, 2015 IEEE International Symposium on (pp. 452-453). IEEE.

Chang, Y., \& Harrington, R. (1977). A surface formulation for characteristic modes of material bodies. Antennas and Propagation, IEEE Transactions on, 25(6), 789-795.

Chang, Y., \& Harrington, R. F. (1974). A surface formulation for characteristic modes of material bodies (Tech. Rep.). DTIC Document.

Chen, C.-C. (1998). Electromagnetic resonances of immersed dielectric spheres. Antennas and Propagation, IEEE Transactions on, 46(7), 1074-1083.

Chen, Y., \& Wang, C. F. (2015). Characteristic modes: Theory and applications in antenna engineering. Hoboken, NJ: John Wiley.

Chen, Y., \& Wang, C.-F. (2014). Surface integral equation based characteristic mode formulation for dielectric resonators. In Antennas and Propagation Society International Symposium (APSURSI) (pp. 846-847). IEEE.

Conwell, P. R., Barber, P. W., \& Rushforth, C. K. (1984). Resonant spectra of dielectric spheres. Journal of the Optical Society of America, 1(1), 62-67.

Dettmann, C., Morozov, G., Sieber, M., \& Waalkens, H. (2009). Internal and external resonances of dielectric disks. EPL (Europhysics Letters), 87(3), 34003. IOP.

FEKO Suite 7.0. EM Software \& Systems - S.A., Stellenbosch, South Africa, http://www.feko.info.

Garbacz, R. J. (1968). A generalized expansion for radiated and scattered fields (PhD thesis). Columbus: The Ohio State University.

Glisson, A. W., Kajfez, D., \& James, J. (1983). Evaluation of modes in dielectric resonators using a surface integral equation formulation. IEEE transactions on Microwave Theory and Techniques, 31(12), 1023-1029.

Harrington, R., \& Mautz, J. (1971). Theory of characteristic modes for conducting bodies. Antennas and Propagation, IEEE Transactions on, 19(5), 622-628.

Harrington, R., Mautz, J., \& Chang, Y. (1972). Characteristic modes for dielectric and magnetic bodies. Antennas and Propagation, IEEE Transactions on, 20(2), 194-198.

Hu, F.-G., \& Wang, C. F. (2016). Integral equation formulations for characteristic modes of dielectric and magnetic bodies. IEEE Transactions on Antennas and Propagation, 64(11), 4770-4776.

Kishk, A. A., Glisson, A., \& Kajfez, D. (1993). Computed resonant frequency and far fields of isolated dielectric discs. In Antennas and Propagation Society International Symposium, 1993. AP-S. Digest (pp. 408-411). IEEE. 
Kotlyar, V., Kovalev, A., \& Kozlov, D. (2016). Calculating the resonance radius of a dielectric cylinder under illumination by a plane TE-wave. Optik-International Journal for Light and Electron Optics, 127(8), 3803-3808.

Larison, P. D. (1989). Evaluation of system identification algorithms for aspect-independent radar target classification (Tech. Rep.). DTIC Document.

Lee, W., Sarkar, T. K., Moon, H., Lamperez, A. G., \& Salazar-Palma, M. (2013). Effect of material parameters on the resonant frequencies of a dielectric object. IEEE Antennas and Wireless Propagation Letters, 12, 1311-1314.

Mie, G. (1908). Beiträge zur optik trüber medien, speziell kolloidaler metallösungen. Annalen der physik, 330(3), $377-445$.

Moser, P. J., Überall, H., \& Morgan, M. A. (1989). Finite element computation of complex resonant frequencies for penetrable axisymmetric bodies. Journal of Electromagnetic Waves and Applications, 3(2), 129-142.

Owen, J., Barber, P., Messinger, B., \& Chang, R. (1981). Determination of optical-fiber diameter from resonances in the elastic scattering spectrum. Optics letters, 6(6), 272-274.

Rayleigh, L. (1918). XLI. the dispersal of light by a dielectric cylinder. The London, Edinburgh, and Dublin Philosophical Magazine and Journal of Science, 36(215), 365-376.

Sarkar, T. K., Mokole, E. L., \& Salazar-Palma, M. (2016). An expose on internal resonance, external resonance, and characteristic modes. IEEE Transactions on Antennas and Propagation, 64(11), 4695-4702.

Van Bladel, J. (1977). Resonant scattering by dielectric cylinders. Microwaves, Optics and Acoustics, IEE Journal on, 1(2), $41-50$. 